\title{
Partial spectral multipliers and partial Riesz transforms for degenerate operators
}

\author{
A.F. M. ter Elst and E. M. Ouhabaz
}

\begin{abstract}
We consider degenerate differential operators of the type $A=$ $-\sum_{k, j=1}^{d} \partial_{k}\left(a_{k j} \partial_{j}\right)$ on $L^{2}\left(\mathbb{R}^{d}\right)$ with real symmetric bounded measurable coefficients. Given a function $\chi \in C_{b}^{\infty}\left(\mathbb{R}^{d}\right)$ (respectively, a bounded Lipschitz domain $\Omega$ ), suppose that $\left(a_{k j}\right) \geq \mu>0$ a.e. on $\operatorname{supp} \chi$ (respectively, a.e. on $\Omega$ ). We prove a spectral multiplier type result: if $F:[0, \infty) \rightarrow \mathbb{C}$ is such that $\sup _{t>0}\|\varphi(.) F(t .)\|_{C^{s}}<\infty$ for some nontrivial function $\varphi \in C_{c}^{\infty}(0, \infty)$ and some $s>d / 2$ then $M_{\chi} F(I+A) M_{\chi}$ is weak type $(1,1)$ (respectively, $P_{\Omega} F(I+A) P_{\Omega}$ is weak type $\left.(1,1)\right)$. We also prove boundedness on $L^{p}$ for all $p \in(1,2]$ of the partial Riesz transforms $M_{\chi} \nabla(I+A)^{-1 / 2} M_{\chi}$. The proofs are based on a criterion for a singular integral operator to be weak type $(1,1)$.
\end{abstract}

\section{Introduction}

Let $A$ be a non-negative self-adjoint uniformly elliptic operator in divergence form. More precisely, let $a_{k j}=a_{j k}: \mathbb{R}^{d} \rightarrow \mathbb{R}$ be bounded measurable functions for all $j, k \in\{1, \ldots, d\}$, and assume that there exists a $\mu>0$ such that

$$
\sum_{k, j=1}^{d} a_{k j}(x) \xi_{k} \xi_{j} \geq \mu|\xi|^{2} \quad \text { for all } \xi=\left(\xi_{1}, \ldots, \xi_{d}\right) \in \mathbb{R}^{d} \text { and } x \in \mathbb{R}^{d}
$$

The operator

$$
A=-\sum_{k, j=1}^{d} \partial_{k}\left(a_{k j} \partial_{j}\right)
$$

defined by quadratic form techniques, is self-adjoint on $L^{2}\left(\mathbb{R}^{d}\right)$. It is a standard fact that $-A$ is the generator of a strongly continuous semigroup $\left(e^{-t A}\right)_{t>0}$ on $L^{2}\left(\mathbb{R}^{d}\right)$.

Mathematics Subject Classification (2010): Primary 42B15; Secondary 45F05.

Keywords: Spectral multipliers, Riesz transforms, singular integral operators, degenerate operators, Gaussian bounds. 
The well-known Aronson estimates assert that $e^{-t A}$ is given by an integral kernel $p_{t}$ (called the heat kernel of $A$ ) which satisfies the Gaussian upper bound:

$$
\left|p_{t}(x, y)\right| \leq C t^{-d / 2} e^{-c|x-y|^{2} / t} \quad \text { for all } t>0 \text { and } x, y \in \mathbb{R}^{d} .
$$

Here $C$ and $c$ are positive constants.

In recent years, harmonic analysis of operators of type $A$ has attracted a lot of attention and substantial progress has been made, in which upper bounds for the heat kernel play a fundamental role. We mention for example the theory of Hardy and BMO spaces associated with such operators (see for example [11] and [16]), spectral multipliers $([10])$ and Riesz transforms (see [8], [2], [18], [20] and the references therein). Concerning spectral multipliers, it is known that if $F:[0, \infty) \rightarrow \mathbb{C}$ is a bounded measurable function then the operator $F(A)$, which is well defined on $L^{2}$ by spectral theory, extends to a bounded operator on $L^{p}$ for all $1<p<\infty$ provided $F$ satisfies the condition

$$
\sup _{t>0}\|\varphi(.) F(t .)\|_{C^{s}}<\infty
$$

for some $s>d / 2$ and some nontrivial auxiliary function $\varphi \in C_{c}^{\infty}(0, \infty)$. See Duong-Ouhabaz-Sikora [10], where a more general result is proved. Note that condition (1.3) is satisfied if $F$ has $[d / 2]+1$ derivatives such that

$$
\sup _{\lambda>0} \lambda^{k}\left|F^{(k)}(\lambda)\right|<\infty \quad \text { for all } k \in\{0,1, \ldots,[d / 2]+1\} .
$$

As an example, one obtains polynomial estimates on $L^{p}$ for imaginary powers of type $\left\|A^{i s}\right\|_{p \rightarrow p} \leq C(1+|s|)^{\beta_{p}}$ for all $\beta_{p}>d|1 / 2-1 / p|$. Taking $F(\lambda):=(1-\lambda / R)_{+}^{\alpha}$, one obtains Bochner-Riesz summability for all $\alpha>d / 2$.

Concerning Riesz transforms $\mathcal{R}_{k}:=\partial_{k} A^{-1 / 2}$, it is an obvious consequence of the ellipticity assumption (1.1) that $\mathcal{R}_{k}$ is bounded on $L^{2}\left(\mathbb{R}^{d}\right)$ for all $k \in\{1, \ldots, d\}$. As for multiplier results, the Gaussian bound (1.2), combined with recent developments on singular integral operators, make it possible to prove that $\mathcal{R}_{k}$ is bounded on $L^{p}\left(\mathbb{R}^{d}\right)$ for all $p \in(1,2)$ with only assumptions (1.1) and bounded measurable coefficients (see Duong-McIntosh [8], Auscher [2], Ouhabaz [18]). Under weak regularity assumption on the coefficients one obtains boundedness of $\mathcal{R}_{k}$ on $L^{p}\left(\mathbb{R}^{d}\right)$ for all $p \in(2, \infty)$ (cf. Auscher [2], Shen [20]).

In the present paper we wish to study similar problems for degenerate operators. Instead of (1.1) we merely assume that

$$
\sum_{k, j=1}^{d} a_{k j}(x) \xi_{k} \xi_{j} \geq 0 \quad \text { for all } \xi=\left(\xi_{1}, \ldots, \xi_{d}\right) \in \mathbb{R}^{d} \text { and } x \in \mathbb{R}^{d}
$$

In this case, we define the form

$$
\mathfrak{a}_{0}(u, v)=\sum_{k, j=1}^{d} \int_{\mathbb{R}^{d}} a_{k j}\left(\partial_{j} u\right)\left(\partial_{k} v\right)
$$


with form domain $D\left(\mathfrak{a}_{0}\right)=C_{c}^{\infty}\left(\mathbb{R}^{d}\right)$. If this form is closable, then $A$ will be the selfadjoint operator associated with its closure. If not, we take the regular part and consider $A$ as the operator associated with the closure of this regular part (see [21] and $[1])$.

Proving results like the previous ones for these operators seems unattainable because Gaussian (or Poisson) upper bounds are not true in general. Even the $L^{1}-L^{\infty}$ estimates of $e^{-t A}$ are not valid in general. What we will do is to restrict the operators to parts where the matrix $\left(a_{k j}\right)$ is elliptic. It is proved by ter Elst and Ouhabaz [12] that if $\chi \in C_{\mathrm{b}}^{\infty}\left(\mathbb{R}^{d}\right)$ and $\mu>0$ are such that $\left(a_{k j}(x)\right) \geq \mu I$ for a.e. $x \in \operatorname{supp} \chi$, then $M_{\chi} e^{-t A} M_{\chi}$ has a Hölder continuous kernel $K_{t}$ which satisfies the Gaussian bound

$$
\left|K_{t}(x, y)\right| \leq C t^{-d / 2} e^{-c|x-y|^{2} / t}(1+t)^{d / 2} \quad \text { for all } t>0 \text { and } x, y \in \mathbb{R}^{d} .
$$

Here $M_{\chi}$ is the operator of multiplication by $\chi$. The same result holds for $P_{\Omega} e^{-t A} P_{\Omega}$ if $\Omega$ is a bounded Lipschitz domain such that $\left(a_{k j}(x)\right) \geq \mu I$ for a.e. $x \in \Omega$ for some $\mu>0$. Here $P_{\Omega}$ is the operator of multiplication by the indicator function $\mathbb{1}_{\Omega}$ of $\Omega$.

Note that in general one cannot get rid of the extra term $(1+t)^{d / 2}$ in the right hand side of (1.6). For example, if $a_{k j}=\delta_{k j}$ on a smooth bounded domain $\Omega$, then $A$ is the Neumann Laplacian on $L^{2}(\Omega)$ and 0 on $L^{2}\left(\mathbb{R}^{d} \backslash \Omega\right)$. It is then clear that the Gaussian bound is not valid without the additional term $(1+t)^{d / 2}$. Because of that additional term in (1.6), we shall consider in the sequel $I+A$ instead of $A$ (of course, one can take $\varepsilon I+A$ for any $\varepsilon>0$ to absorb the factor $\left.(1+t)^{d / 2}\right)$.

For spectral multipliers and Riesz transforms we will prove the following results. Suppose that $\chi \in C_{\mathrm{b}}^{\infty}\left(\mathbb{R}^{d}\right)$ (resp., a bounded Lipschitz domain $\Omega$ ) is such that $\left(a_{k j}(x)\right) \geq \mu I$ for a.e. $x \in \operatorname{supp} \chi$ (resp., for a.e. $x \in \Omega$ ) for some constant $\mu>0$. The main theorems of this paper are the following:

Theorem 1.1. Let $F:[0, \infty) \rightarrow \mathbb{C}$ be a bounded function such that

$$
\sup _{t>0}\|\varphi(.) F(t .)\|_{C^{s}}<\infty
$$

for some $s>d / 2$ and some nontrivial function $\varphi \in C_{c}^{\infty}(0, \infty)$. Then the operator $M_{\chi} F(I+A) M_{\chi}$ (resp., $\left.P_{\Omega} F(I+A) P_{\Omega}\right)$ is bounded on $L^{p}\left(\mathbb{R}^{d}\right)$ for all $1<p<\infty$.

Theorem 1.2. The Riesz transform type operator $M_{\chi} \partial_{k}(I+A)^{-1 / 2} M_{\chi}$ is bounded on $L^{p}\left(\mathbb{R}^{d}\right)$ for all $1<p \leq 2$ and $k \in\{1, \ldots, d\}$.

Now we discuss how we prove these results. In the elliptic case, besides the Gaussian bound (1.2), the proof of the boundedness of the spectral multipliers or Riesz transforms rely on a criterion proved by Duong and McIntosh [9] for singular integral operators to be weak type $(1,1)$. This criterion says that if $T$ is bounded on $L^{2}$ with a (singular) kernel $K$ such that there exists a family of operators $\left(A_{t}\right)_{t>0}$ given by integral kernels $a_{t}$ which satisfy Gaussian (or Poisson) bounds, $T A_{t}$ is also given by a (singular) kernel $K_{t}$ and there are $C, \delta>0$ such that

$$
\int_{|x-y| \geq \delta \sqrt{t}}\left|K(x, y)-K_{t}(x, y)\right| d x \leq C
$$


for all $t>0$ and a.e. $y$, then $T$ is weak type $(1,1)$. In applications to spectral multipliers of elliptic operators we start with $T=F(A)$ and one takes $A_{t}=e^{-t A}$. Therefore, $K_{t}$ is the kernel of the operator $F(A) e^{-t A}$ which can be seen as a regularization of $F(A)$. In the degenerate case and because of (1.6), it is tempting to choose $A_{t}=M_{\chi} e^{-t(I+A)} M_{\chi}$. Then,

$$
T A_{t}=M_{\chi} F(I+A) M_{\chi} M_{\chi} e^{-t(I+A)} M_{\chi}=M_{\chi} F(I+A) M_{\chi}^{2} e^{-t(I+A)} M_{\chi} .
$$

Now, the presence of $M_{\chi}^{2}$ makes it imposible to regularize $F(I+A)$ by $e^{-t(I+A)}$. The simple fact that we do not have $F(I+A)$ next to $e^{-t(I+A)}$ in the expression for $T A_{t}$ destroys this strategy. The same problem occurs for the truncated Riesz transform $M_{\chi} \partial_{k}(I+A)^{-1 / 2} M_{\chi}$. To overcome this difficulty we prove a version of the Duong-McIntosh criterion that is suitable for our purpose. It reads as follows (see Theorems 2.1 and 2.3 together with Remark 2.2 for precise and quantitative statements).

Theorem 1.3. Let $T$ be a bounded linear operator on $L^{2}$ and $\left(A_{t}\right)_{t>0}$ a family of linear operators which satisfy $L^{1}-L^{2}$ off-diagonal estimates. Suppose that there exists a bounded linear operator $S$ on $L^{2}$ and $\delta, W>0$ such that

$$
\int_{|x-y| \geq(1+\delta) t}\left|\left(T-S A_{t}\right) u(y)\right| d y \leq W\|u\|_{1}
$$

for all $x \in \mathbb{R}^{d}, t>0$ and $u \in L^{1} \cap L^{\infty}$ supported in the ball $B(x, t)$. Then $T$ is weak type $(1,1)$.

Note that the estimate (1.8) is satisfied if $T$ and $S A_{t}$ are given by (singular) kernels $K$ and $K_{t}$ and there are $C, \delta>0$ such that

$$
\int_{|x-y| \geq \delta \sqrt{t}}\left|K(x, y)-K_{t}(x, y)\right| d x \leq C
$$

for all $t>0$ and a.e. $y \in \mathbb{R}^{d}$.

Theorem 1.3 gives the extra freedom to choose any appropriate operator $S$, which not need equal $T$. Returning to spectral multipliers for degenerate operators $A$, we had $T=M_{\chi} F(I+A) M_{\chi}$ and we choose now $S=M_{\chi} F(I+A)$ and $A_{t}=e^{-t(I+A)} M_{\chi}$. Then $T A_{t}=M_{\chi} F(A+I) e^{-t(I+A)} M_{\chi}$ for which we can prove the estimate in Theorem 1.3. Similarly for the Riesz transforms where $T=M_{\chi} \partial_{k}(I+A)^{-1} M_{\chi}$, we take $S=M_{\chi} \partial_{k}(I+A)^{-1}$ which turns out to be bounded on $L^{2}$ and $A_{t}=e^{-t(I+A)} M_{\chi}$. We emphasize that $A_{t}=e^{-t(I+A)} M_{\chi}$ satisfies the usual $L^{1}-L^{2}$ off-diagonal estimates but it is not known whether it satisfies Gaussian upper bounds in general ${ }^{1}$. We believe that our version of the Duong-McIntosh criterion can be used in other circumstances in which products of several operators come into play. Also, as in [9], our version holds for operators on domains of spaces of homogeneous type.

\footnotetext{
${ }^{1}$ Under the additional assumption that $a_{k j} \in W^{1, \infty}\left(\mathbb{R}^{d}\right)$, we proved recently in [13] that $e^{-t(I+A)} M_{\chi}$ has a kernel which satisfies a Gaussian bound.
} 
Notation. We fix some notation which we will use throughout this paper. If $(X, \rho, \mu)$ is a metric measure space, $x \in X, r>0$ and $j \in \mathbb{N}$, then we denote by $B(x, r):=\{y \in X: \rho(x, y)<r\}$ the open ball of $X$ with centre $x$ and radius $r$, by $C_{j}(x, r)$ the annulus $B\left(x, 2^{j+1} r\right) \backslash B\left(x, 2^{j} r\right)$ if $j \geq 2$, and by $C_{1}(x, r)$ the ball $B(x, 4 r)$. Let $v(x, r)=\mu(B(x, r))$ be the volume of the ball $B(x, r)$. Next, $\|T\|_{p \rightarrow q}$ is the norm of $T$ as an operator from $L^{p}$ to $L^{q}$. If $E$ is a measurable set, then $P_{E}$ denotes the operator of multiplication by the indicator function $\mathbb{1}_{E}$ of $E$. If $s \in$ $(0, \infty) \backslash \mathbb{N}$, we denote by $C^{s}$ the space of all Lipschitz functions on $[0, \infty)$ of order $s$ (i.e., functions that are continuously differentiable up to $[s]$ and for which the derivative of order $[s]$ is Hölder continuous of order $s-[s]$ ). By $W^{r, p}$ we denote the classical Sobolev spaces on $\mathbb{R}^{d}$.

All our operators are linear operators.

We emphasize that we shall use $C, C^{\prime}, c, \ldots$ for all inessential constants. A constant $C$ may differ from line to line, even within one line.

\section{Singular integral operators}

Let $(X, \mu, \rho)$ be a metric measure space. We shall assume that $0<v(x, r)<\infty$ for all $x \in X$ and $r>0$, and that $X$ is a space of homogeneous type. This means that it satisfies the doubling condition

$$
v(x, 2 r) \leq C_{0} v(x, r)
$$

for some $C_{0}>0$, uniformly for all $x \in X$ and $r>0$. If (2.1) is satisfied then there exist positive constants $C_{1}$ and $d$ such that

$$
v(x, \lambda r) \leq C_{1} \lambda^{d} v(x, r)
$$

for all $x \in X$ and $r \geq 1$. Let $\Omega$ be an open subset of $X$. It is endowed with $\rho$ and $\mu$ but $(\Omega, \mu, \rho)$ is not necessarily a space of homogeneous type. Let $T$ be a bounded linear operator on $L^{p_{0}}(\Omega):=L^{p_{0}}(\Omega, \mu)$ for some $p_{0} \in[1, \infty)$. We say that $T$ is given by a kernel $K: \Omega \times \Omega \rightarrow \mathbb{C}$ if $K$ is measurable and

$$
T u(x)=\int_{\Omega} K(x, y) u(y) d \mu(y)
$$

for all $u \in L^{p_{0}}(\Omega)$ with bounded support and a.e. $x$ outside the support of $u$. We also say that $K$ is the associated kernel of $T$. A classical problem in harmonic analysis is to find conditions on the kernel $K$ under which the operator $T$ can be extended from $L^{p_{0}}(\Omega)$ to other $L^{p}(\Omega)$-spaces. Several results are known in this direction. We refer the reader to [22], [9], [3], [2] and the references therein.

The main result in [9] states that if there exists a family of bounded operators $A_{t}$, with $t>0$, which are given by integral kernels $a_{t}$ satisfying a Gaussian or Poisson estimate and if the associated kernel of $T-T A_{t}$ does not oscillate too much in a certain sense then $T$ is weak type $(1,1)$. Here we prove by the same method that if there exists a bounded operator $S$ on $L^{q_{0}}(X)$ for some $q_{0} \in(1, \infty)$ such that the associated kernel of $T-S A_{t}$ does not oscillate too much then $T$ is weak type $(1,1)$. 
As explained in the introduction, this new version gives the extra freedom to choose any appropriate $S$ which need not coincide with $T$. This extension turns out to be powerful for proving spectral multiplier type results as well as Riesz transforms for degenerate operators, whereas it is not clear how to apply the condition from [9]. Note also that, following ideas from [3] and [2] we can weaken the assumption on the kernel of $A_{t}$. Instead of assuming a Gaussian or Poisson bound, we merely assume an $L^{1}-L^{q_{0}}$ off-diagonal estimate (see (2.4) below). This difference is again illustrated in our application to degenerate operators. In addition it is possible to formulate the result in [9] without reference to the kernels (see also the remark immediately after the next theorem).

We first state and prove the result in the case $\Omega=X$.

Theorem 2.1. Let $T$ be a nonzero bounded linear operator on $L^{p_{0}}(X)$ for some $p_{0} \in(1, \infty)$. Suppose that there exists a bounded linear operator $S$ on $L^{q_{0}}(X)$ for some $q_{0} \in(1, \infty)$, a family of bounded linear operators $\left(A_{t}\right)_{t>0}$ on $L^{q_{0}}(X)$ and a sequence $(g(j))_{j \in \mathbb{N}}$ in $\mathbb{R}$ such that

$$
\left(\frac{1}{v\left(x, 2^{j+1} t\right)} \int_{C_{j}(x, t)}\left|A_{t} f\right|^{q_{0}}\right)^{1 / q_{0}} \leq g(j) \frac{1}{v(x, t)} \int_{B(x, t)}|f|
$$

for all $x \in X, t>0, j \in \mathbb{N}$ and $f \in L^{q_{0}}(B(x, t))$, and $\sum_{j=1}^{\infty} 2^{j d} g(j)<\infty$. Finally, suppose there exist $\delta, W>0$ such that

$$
\int_{X \backslash B(x,(1+\delta) t)}\left|\left(T-S A_{t}\right) u\right| \leq W\|u\|_{1}
$$

for all $x \in X, t>0$ and $u \in L^{1}(X) \cap L^{\infty}(X)$ supported in the ball $B(x, t)$. Then $T$ is a weak type $(1,1)$ operator with

$$
\|T\|_{L^{1} \rightarrow L^{1, w}} \leq C(1+\delta)^{d}\left(W+\|T\|_{p_{0} \rightarrow p_{0}}+\|S\|_{q_{0} \rightarrow q_{0}}^{q_{0}}\|T\|_{p_{0} \rightarrow p_{0}}^{1-q_{0}}\right) .
$$

Here $C$ is a constant depending only on the constants in (2.2). In particular, $T$ extends to a bounded operator on $L^{p}(X)$ for all $p \in\left(1, p_{0}\right)$.

Remark 2.2. Let $p_{0}, q_{0} \in(1, \infty), T \in \mathcal{L}\left(L^{p_{0}}(X)\right)$, and for all $t>0$, let $S, A_{t} \in$ $\mathcal{L}\left(L^{q_{0}}(X)\right)$. Suppose that $T$ and $S A_{t}$ have kernels $K$ and $K_{t}$, respectively. Let $\delta, W>0$ and assume that

$$
\int_{\rho(x, y) \geq \delta t}\left|K(x, y)-K_{t}(x, y)\right| d \mu(x) \leq W<\infty,
$$

for all $t>0$ and $y \in X$. Fix now $x \in X, t>0$ and $u \in L^{1}(X) \cap L^{\infty}(X)$ supported in the ball $B(x, t)$. Then

$$
\begin{aligned}
\int_{X \backslash B(x,(1+\delta) t)}\left|\left(T-S A_{t}\right) u(y)\right| d \mu(y) \\
\quad=\int_{X \backslash B(x,(1+\delta) t)}\left|\int_{B(x, t)}\left(K(y, z)-K_{t}(y, z)\right) u(z) d \mu(z)\right| d \mu(y) \\
\leq \int_{X} \int_{\rho(y, z) \geq \delta t}\left|K(y, z)-K_{t}(y, z)\right| d \mu(y)|u(z)| d \mu(z) \leq W\|u\|_{1} .
\end{aligned}
$$


Thus, (2.5) is satisfied. The condition (2.7) is the direct analogue of the condition in Duong-McIntosh [9].

We also observe that one does not need kernels for both operators $T$ and $T A_{t}$, rather only a kernel $H_{t}(x, y)$ for the difference $T-S A_{t}$. We may then replace $K(x, y)-K_{t}(x, y)$ in $(2.7)$ by $H_{t}(x, y)$. On the other hand, the use of the local estimate (2.5), which does not appeal to kernels, may have the advantage of avoiding issues of the measurability with respect to $x$ and $y$ of the expected singular kernels.

Proof. As mentioned before, the arguments are similar to those used in [9] and [2]. We give the details for convenience. Recall we denote by $C$ all inessential constants.

We begin with the classical Calderón-Zygmund decomposition. There exist $c, N>0$ such that the following is valid. Fix $f \in L^{1}(X) \cap L^{\infty}(X)$ and $\alpha>$ $\|f\|_{1} / \mu(X)$. There exist $g, b_{1}, b_{2}, \ldots \in L^{1}(X) \cap L^{\infty}(X)$ such that

$$
f=g+b=g+\sum_{i} b_{i}
$$

and

(i) $|g(x)| \leq c \alpha$ for a.e. $x \in X$,

(ii) each $b_{i}$ is supported in a ball $B_{i}=B\left(x_{i}, r_{i}\right)$ and $\left\|b_{i}\right\|_{1} / v\left(x_{i}, r_{i}\right) \leq c \alpha$,

(iii) $\sum_{i} v\left(x_{i}, r_{i}\right) \leq c\|f\|_{1} / \alpha$, and,

(iv) there exists a constant $N$ such that $\sum_{i} \mathbb{1}_{B_{i}}(x) \leq N$ for a.e. $x \in X$.

See Section III.2 in [5].

We proceed in several steps.

Step 1. Using the boundedness of $T$ on $L^{p_{0}}$ we have

$$
\begin{aligned}
\mu(\{x \in X:|(T g)(x)|>\alpha\}) & \leq \frac{\|T\|_{p_{0} \rightarrow p_{0}}^{p_{0}}}{\alpha^{p_{0}}}\|g\|_{p_{0}}^{p_{0}} \\
& \leq C \alpha^{p_{0}-1} \frac{\|T\|_{p_{0} \rightarrow p_{0}}^{p_{0}}}{\alpha^{p_{0}}}\|g\|_{1} \leq C \frac{\|T\|_{p_{0} \rightarrow p_{0}}^{p_{0}}}{\alpha}\|g\|_{1} .
\end{aligned}
$$

It follows from (ii) and (iii) above that $\|b\|_{1} \leq c\|f\|_{1}$ and hence $\|g\|_{1} \leq(1+c)\|f\|_{1}$. Therefore,

$$
\mu(\{x \in X:|(T g)(x)|>\alpha\}) \leq C \frac{\|T\|_{p_{0} \rightarrow p_{0}}^{p_{0}}}{\alpha}\|f\|_{1}
$$

Step 2. We shall prove that

$$
\left\|\sum_{i} A_{r_{i}} b_{i}\right\|_{q_{0}} \leq C \alpha^{1-1 / q_{0}}\|f\|_{1}^{1 / q_{0}} .
$$


We use arguments similar to those in [2]. Fix $u \in L^{q_{0}^{\prime}}$ with $\|u\|_{q_{0}^{\prime}}=1$, where $q_{0}^{\prime}$ is the dual exponent of $q_{0}$. Let $i, j \in \mathbb{N}$ and set $C_{i, j}:=C_{j}\left(x_{i}, r_{i}\right)$. Then

$$
\begin{aligned}
\int_{C_{i, j}}\left|A_{r_{i}} b_{i}\right||u| & \leq\left(\int_{C_{i, j}}\left|A_{r_{i}} b_{i}\right|^{q_{0}}\right)^{1 / q_{0}}\left(\int_{C_{i, j}}|u|^{q_{0}^{\prime}}\right)^{1 / q_{0}^{\prime}} \\
& \leq g(j) \frac{v\left(x_{i}, 2^{j+1} r_{i}\right)^{1 / q_{0}}}{v\left(x_{i}, r_{i}\right)}\left(\int\left|b_{i}\right|\right)\left(\int_{C_{i, j}}|u|^{q_{0}^{\prime}}\right)^{1 / q_{0}^{\prime}} \\
& \leq \operatorname{c\alpha g} g(j) v\left(x_{i}, 2^{j+1} r_{i}\right)\left(\frac{1}{v\left(x_{i}, 2^{j+1} r_{i}\right)} \int_{C_{i, j}}|u|^{q_{0}^{\prime}}\right)^{1 / q_{0}^{\prime}}
\end{aligned}
$$

where we have used (2.4) and property (ii) in the Calderón-Zygmund decomposition. Denote by $\mathcal{M}$ the Hardy-Littlewood maximal operator. Then

$$
\frac{1}{v\left(x_{i}, 2^{j+1} r_{i}\right)} \int_{C_{i, j}}|u|^{q_{0}^{\prime}} \leq \mathcal{M}\left(|u|^{q_{0}^{\prime}}\right)(y)
$$

for all $y \in B_{i}$. Combining the previous inequalities and using the doubling condition (2.2) one estimates

$$
\int_{C_{i, j}}\left|A_{r_{i}} b_{i}\right||u| \leq C \alpha 2^{j d} g(j) v\left(x_{i}, r_{i}\right)\left(\mathcal{M}\left(|u|^{q_{0}^{\prime}}\right)(y)\right)^{1 / q_{0}^{\prime}} .
$$

Taking the integral over $y \in B_{i}$ gives

$$
\int_{C_{i, j}}\left|A_{r_{i}} b_{i}\right||u| \leq C \alpha 2^{j d} g(j) \int_{B_{i}}\left(\mathcal{M}\left(|u|^{q_{0}^{\prime}}\right)(y)\right)^{1 / q_{0}^{\prime}} d \mu(y) .
$$

We sum over $j$ and $i$ and use $\sum_{j} 2^{j d} g(j)<\infty$ together with property (iv) in the Calderón-Zygmund decomposition to obtain

$$
\begin{aligned}
& \int_{X}\left|\sum_{i} A_{r_{i}} b_{i}\right||u| \leq C \alpha \int_{X} \mathbb{1}_{\cup_{i} B_{i}}(y)\left(\mathcal{M}\left(|u|^{q_{0}^{\prime}}\right)(y)\right)^{1 / q_{0}^{\prime}} d \mu(y) \\
& \leq C \alpha\left\|\mathbb{1}_{\cup_{i} B_{i}}\right\|_{q_{0}}\left\|\left(\mathcal{M}\left(|u|^{q_{0}^{\prime}}\right)\right)^{1 / q_{0}^{\prime}}\right\|_{q_{0}^{\prime}, w} \leq C \alpha\left(\sum_{i} v\left(x_{i}, r_{i}\right)\right)^{1 / q_{0}}\left\||u|^{q_{0}^{\prime}}\right\|_{1}^{1 / q_{0}^{\prime}} .
\end{aligned}
$$

Note that we have used the fact that $\mathcal{M}$ is weak type $(1,1)$ to obtain the last inequality. Using now (iii) of the Calderón-Zygmund decomposition and $\|u\|_{q_{0}^{\prime}}=1$, we obtain (2.9).

By assumption, $S$ is bounded on $L^{q_{0}}$. Hence

$$
\mu\left(\left\{x \in X:\left|\left(S \sum_{i} A_{r_{i}} b_{i}\right)(x)\right|>\alpha\right\}\right) \leq \frac{1}{\alpha^{q_{0}}}\|S\|_{q_{0} \rightarrow q_{0}}^{q_{0}}\left\|\sum_{i} A_{r_{i}} b_{i}\right\|_{q_{0}}^{q_{0}} .
$$

Now we use (2.9) to obtain

$$
\mu\left(\left\{x \in X:\left|\left(S \sum_{i} A_{r_{i}} b_{i}\right)(x)\right|>\alpha\right\}\right) \leq \frac{C}{\alpha}\|S\|_{q_{0} \rightarrow q_{0}}^{q_{0}}\|f\|_{1} .
$$


Step 3. Let $\delta$ be as in (2.5) and for all $i \in \mathbb{N}$ set $Q_{i}:=B\left(x_{i},(1+\delta) r_{i}\right)$, the ball of centre $x_{i}$ and radius $(1+\delta) r_{i}$. Then

$$
\begin{aligned}
\mu(\{x \in X & \left.\left.:\left|\sum_{i}\left(T-S A_{r_{i}}\right) b_{i}(x)\right|>\alpha\right\}\right) \\
& \leq \sum_{i} \mu\left(Q_{i}\right)+\mu\left(\left\{x \in X \backslash \bigcup_{j} Q_{j}:\left|\sum_{i}\left(\left(T-S A_{r_{i}}\right) b_{i}\right)(x)\right|>\alpha\right\}\right) \\
& \leq C(1+\delta)^{d} \sum_{i} v\left(x_{i}, r_{i}\right)+\frac{1}{\alpha} \int_{X \backslash \bigcup_{j} Q_{j}}\left|\sum_{i}\left(\left(T-S A_{r_{i}}\right) b_{i}\right)(x)\right| d \mu(x) \\
& \leq \frac{C(1+\delta)^{d}}{\alpha}\|f\|_{1}+\frac{1}{\alpha} \sum_{i} \int_{X \backslash Q_{i}}\left|\left(\left(T-S A_{r_{i}}\right) b_{i}\right)(x)\right| d \mu(x) \\
& \leq \frac{C(1+\delta)^{d}}{\alpha}\|f\|_{1}+\frac{W}{\alpha} \sum_{i} \int_{\mid}\left|b_{i}(y)\right| d \mu(y) \\
& \leq \frac{C(1+\delta)^{d}(1+W)}{\alpha}\|f\|_{1} .
\end{aligned}
$$

Note that the penultimate inequality follows from assumption (2.5) and the last one from properties (ii) and (iii) in the Calderón-Zygmund decomposition. Hence

$$
\mu\left(\left\{x \in X:\left|\sum_{i}\left(\left(T-S A_{r_{i}}\right) b_{i}\right)(x)\right|>\alpha\right\}\right) \leq \frac{C(1+\delta)^{d}(1+W)}{\alpha}\|f\|_{1} .
$$

Step 4. It follows from (2.8) that

$$
\begin{aligned}
\mu(\{x \in X: \mid & (T f)(x) \mid>\alpha\}) \\
& \leq \mu(\{x \in X:|(T g)(x)|>\alpha / 2\})+\mu(\{x \in X:|(T b)(x)|>\alpha / 2\}) \\
& \leq C \frac{\|T\|_{p_{0} \rightarrow p_{0}}^{p_{0}}}{\alpha}\|f\|_{1}+\mu(\{x \in X:|(T b)(x)|>\alpha / 2\}) .
\end{aligned}
$$

For the second term we use (2.10) and (2.11) to estimate

$$
\begin{aligned}
\mu(\{x \in X & :|(T b)(x)|>\alpha / 2\}) \\
= & \mu\left(\left\{x \in X:\left|\sum_{i}\left(S A_{r_{i}} b_{i}\right)(x)+\sum_{i}\left(\left(T-S A_{r_{i}}\right) b_{i}\right)(x)\right|>\alpha / 2\right\}\right) \\
\leq & \mu\left(\left\{x \in X:\left|\left(S \sum_{i} A_{r_{i}} b_{i}\right)(x)\right|>\alpha / 4\right\}\right) \\
& +\mu\left(\left\{x \in X:\left|\sum_{i}\left(\left(T-S A_{r_{i}}\right) b_{i}\right)(x)\right|>\alpha / 4\right\}\right) \\
\leq & \frac{C(1+\delta)^{d}}{\alpha}\left(\|S\|_{q_{0} \rightarrow q_{0}}^{q_{0}}+(1+W)\right)\|f\|_{1} .
\end{aligned}
$$

We then conclude that $T$ is of weak type $(1,1)$ with a weak type estimate

$$
\|T\|_{L^{1} \rightarrow L^{1, w}} \leq C(1+\delta)^{d}\left(1+W+\|T\|_{p_{0} \rightarrow p_{0}}^{p_{0}}+\|S\|_{q_{0} \rightarrow q_{0}}^{q_{0}}\right) .
$$


Replacing $T$ and $S$ by $\|T\|_{p_{0} \rightarrow p_{0}}^{-1} T$ and $\|T\|_{p_{0} \rightarrow p_{0}}^{-1} S$, we obtain (2.5) with $\|T\|_{p_{0} \rightarrow p_{0}}^{-1} W$ instead of $W$. Thus applying (2.12) to

$$
\|T\|_{p_{0} \rightarrow p_{0}}^{-1} T, \quad\|T\|_{p_{0} \rightarrow p_{0}}^{-1} S \text { and }\|T\|_{p_{0} \rightarrow p_{0}}^{-1} W
$$

yields (2.6).

Finally, by the Marcinkiewicz interpolation theorem the operator $T$ extends to a bounded operator from $L^{p}(X) \cap L^{p_{0}}(X)$ to $L^{p}(X)$ for all $p \in\left(1, p_{0}\right)$.

Following again an idea in [9] we can prove a version of the previous theorem on arbitrary domains. Let $\Omega$ be an open subset of $X$ and assume that $T$ is bounded on $L^{p_{0}}(\Omega)$ and $S$ and $A_{t}$ are bounded on $L^{q_{0}}(\Omega)$. We define $\widetilde{T}: L^{p_{0}}(X) \rightarrow L^{p_{0}}(X)$ by

$$
\widetilde{T} f=\mathbb{1}_{\Omega} T\left(\mathbb{1}_{\Omega} f\right)
$$

and similarly for $\widetilde{S}$ and $\widetilde{A_{t}}$. If $A_{t}$ satisfies (2.13) below then $\widetilde{A_{t}}$ satisfies $(2.4)$. The operator $T$ is weak type $(1,1)$ if and only if $\widetilde{T}$ is weak type $(1,1)$. Applying the previous theorem to $\widetilde{T}, \widetilde{S}$ and $\widetilde{A_{t}}$ gives the following result.

Theorem 2.3. Let $T$ be a nonzero bounded linear operator on $L^{p_{0}}(\Omega)$ for some $p_{0} \in(1, \infty)$. Suppose there exists a bounded operator $S$ on $L^{q_{0}}(\Omega)$ for some $q_{0} \in$ $(1, \infty)$, a family of bounded operators $\left(A_{t}\right)_{t>0}$ on $L^{q_{0}}(\Omega)$ and a sequence $(g(j))_{j \in \mathbb{N}}$ in $\mathbb{R}$ such that

$$
\left(\frac{1}{v\left(x, 2^{j+1} t\right)} \int_{C_{j}(x, t) \cap \Omega}\left|A_{t} f\right|^{q_{0}}\right)^{1 / q_{0}} \leq g(j) \frac{1}{v(x, t)} \int_{B(x, t) \cap \Omega}|f|
$$

for all $x \in \Omega, t>0, j \in \mathbb{N}, f \in L^{q_{0}}(B(x, t) \cap \Omega)$, and $\sum_{j=1}^{\infty} 2^{j d} g(j)<\infty$. Finally, suppose there exist $\delta, W>0$ such that

$$
\int_{\Omega \backslash B(x,(1+\delta) t)}\left|\left(\left(T-S A_{t}\right) u\right)(y)\right| d \mu(y) \leq W\|u\|_{1}
$$

for all $x \in X, t>0$ and $u \in L^{1}(\Omega) \cap L^{\infty}(\Omega)$ supported in the ball $B(x, t) \cap \Omega$.

Then $T$ is a weak type $(1,1)$ operator with

$$
\|T\|_{L^{1}(\Omega) \rightarrow L^{1, w}(\Omega)} \leq C(1+\delta)^{d}\left(W+\|T\|_{p_{0} \rightarrow p_{0}}+\|S\|_{q_{0} \rightarrow q_{0}}^{q_{0}}\|T\|_{p_{0} \rightarrow p_{0}}^{1-q_{0}}\right) .
$$

Here $C$ is a constant depending only on the constants in (2.2). In particular, $T$ extends to a bounded operator on $L^{p}(\Omega)$ for all $p \in\left(1, p_{0}\right)$.

As in Remark 2.2 the condition (2.14) follows if the operators $T$ and $S A_{t}$ are given by kernels $K$ and $K_{t}$ (in the sense of (2.3)) and there are $\delta, W>0$ such that

$$
\int_{\rho(x, y) \geq \delta t}\left|K(x, y)-K_{t}(x, y)\right| d \mu(x) \leq W<\infty,
$$

for all $t>0$ and $y \in \Omega$. It suffices to note that the associated kernel of $\widetilde{T}$ is the extension by 0 outside $\Omega \times \Omega$ of the kernel of $T$ where $\widetilde{T} f=\mathbb{1}_{\Omega} T\left(\mathbb{1}_{\Omega} f\right)$ as above. Similarly for the kernel of $\widetilde{S A_{t}}$. 
In the previous theorems, we may replace the annulus $C_{j}(x, r)$ by the annulus $A(x, j, r):=B(x,(j+1) r) \backslash B(x, j r)$. In that case, $v\left(x, 2^{j+1} r\right)$ has to be replaced by $v(x,(j+1) r)$ and the condition on $g$ becomes $\sum_{j} j^{d} g(j)<\infty$.

Following [3], it is proved in [2] that a bounded operator $T$ on $L^{2}(X)$ is of weak type $(r, r)$ if

$$
\left(\frac{1}{v\left(x, 2^{j+1} t\right)} \int_{C_{j}(x, t)}\left|T\left(I-A_{t}\right) f\right|^{2}\right)^{1 / 2} \leq g(j)\left(\frac{1}{v(x, t)} \int_{B(x, t)}|f|^{r}\right)^{1 / r}
$$

and

$$
\left(\frac{1}{v\left(x, 2^{j+1} t\right)} \int_{C_{j}(x, t)}\left|A_{t} f\right|^{2}\right)^{1 / 2} \leq g(j)\left(\frac{1}{v(x, t)} \int_{B(x, t)}|f|^{r}\right)^{1 / r}
$$

for all $x \in X, t>0, j \in \mathbb{N}, f \in L^{2}$ supported in $B(x, t)$, and $\sum g(j) 2^{d j}<\infty$. One can prove a version of this result in which $T-T A_{t}$ in $(2.17)$ is replaced by $T-S A_{t}$ as in Theorem 2.1. We do not give the details here since we have no concrete application. Theorem 2.1 is suitable for our purpose.

Finally, let us mention that a Gaussian upper bound implies assumption (2.4). Indeed, assume that $A_{t}$ is given by a kernel $a_{t}$ such that

$$
\left|a_{t}(x, y)\right| \leq \frac{C}{v\left(y, t^{1 / m}\right)} \exp \left\{-c \frac{\rho(x, y)^{m /(m-1)}}{t^{1 /(m-1)}}\right\}
$$

for all $t>0$ and $x, y \in X$. Here $m \geq 2$ and $C, c$ are two positive constants. Fix $x_{0} \in X$. Note that the operator $\mathbb{1}_{C_{j}\left(x_{0}, t\right)} A_{t^{m}} \mathbb{1}_{B\left(x_{0}, t\right)}$ has the kernel given by $(x, y) \mapsto \mathbb{1}_{C_{j}\left(x_{0}, t\right)}(x) a_{t^{m}}(x, y) \mathbb{1}_{B\left(x_{0}, t\right)}(y)$. But

$$
\begin{aligned}
\mid \mathbb{1}_{C_{j}\left(x_{0}, t\right)}(x) & a_{t^{m}}(x, y) \mathbb{1}_{B\left(x_{0}, t\right)}(y) \mid \\
& \leq \frac{C}{v(y, t)} \mathbb{1}_{C_{j}\left(x_{0}, t\right)}(x) \mathbb{1}_{B\left(x_{0}, t\right)}(y) \exp \left\{-c \frac{\rho(x, y)^{m /(m-1)}}{t^{m /(m-1)}}\right\} \\
& \leq \frac{C}{v(y, t)} e^{-c 2^{j m /(m-1)}} \mathbb{1}_{B\left(x_{0}, t\right)}(y) \leq \frac{C}{v\left(x_{0}, t\right)} e^{-c 2^{j m /(m-1)}},
\end{aligned}
$$

where the doubling property was used in the last step. This is an $L^{1}-L^{\infty}$ estimate. By interpolation, it implies an $L^{1}-L^{q_{0}}$ estimate giving $(2.4)$ for every $q_{0} \in(1, \infty)$.

\section{A partial multiplier theorem for degenerate operators}

Let the coefficients $a_{k j}$, form $\mathfrak{a}_{0}$, and the self-adjoint operator $A$ associated with $\mathfrak{a}_{0}$ be as in the introduction. For every bounded measurable function $F:[0, \infty) \rightarrow \mathbb{C}$, the operator $F(A)$ is well defined by spectral theory and is bounded on $L^{2}\left(\mathbb{R}^{d}\right)$. As mentioned in the introduction, if $A$ is uniformly elliptic then $F(A)$ extends to a bounded operator on $L^{p}\left(\mathbb{R}^{d}\right)$ for all $p \in(1, \infty)$ provided $F$ has a finite number of derivatives on $[0, \infty)$ which have good decay. We address here the same problem for degenerate operators. This is a difficult problem because no global Gaussian upper bounds are available for $A$ in general. 
We prove a partial result by projecting on the part where the matrix $\left(a_{k j}\right)$ is uniformly elliptic. There are two versions.

Theorem 3.1. Let $\Omega \subset \mathbb{R}^{d}$ be an open bounded set with Lipschitz boundary. Suppose there exists a $\mu>0$ such that $\left(a_{k j}(x)\right) \geq \mu I$ for almost every $x \in \Omega$ and denote by $P_{\Omega}$ the projection from $L^{2}\left(\mathbb{R}^{d}\right)$ onto $L^{2}(\Omega)$. Set $H=A+I$. Let $F:[0, \infty) \rightarrow \mathbb{C}$ be a bounded function such that

$$
\sup _{t>0}\|\varphi(.) F(t .)\|_{C^{s}}<\infty
$$

for some $s>d / 2$ and some nontrivial function $\varphi \in C_{c}^{\infty}(0, \infty)$. Then $P_{\Omega} F(H) P_{\Omega}$ is of weak type $(1,1)$ and extends to a bounded operator on $L^{p}\left(\mathbb{R}^{d}\right)$ for all $p \in(1, \infty)$.

Theorem 3.2. Let $\chi \in C_{\mathrm{b}}^{\infty}\left(\mathbb{R}^{d}\right), \mu>0$ and suppose that $\left(a_{k j}(x)\right) \geq \mu I$ for almost every $x \in \operatorname{supp} \chi$. Set $H=A+I$. Let $F:[0, \infty) \rightarrow \mathbb{C}$ be a bounded function such that

$$
\sup _{t>0}\|\varphi(.) F(t .)\|_{C^{s}}<\infty
$$

for some $s>d / 2$ and some nontrivial function $\varphi \in C_{c}^{\infty}(0, \infty)$. Then $M_{\chi} F(H) M_{\chi}$ is of weak type $(1,1)$ and extends to a bounded operator on $L^{p}\left(\mathbb{R}^{d}\right)$ for all $p \in(1, \infty)$.

The proofs of both theorems are almost the same. They rely mainly on weighted estimates for the associated kernel of $M_{\chi} F(H) M_{\chi}$ (or the kernel of $P_{\Omega} F(H) P_{\Omega}$ ), together with Theorem 2.1. The proof of weighted estimates for the kernel of $M_{\chi} F(H) M_{\chi}$ (or of $P_{\Omega} F(H) P_{\Omega}$ ) is based on partial Gaussian bounds proved in [12] and a similar strategy as in [10] and [18].

In the rest of this section we assume that there exists a constant $\mu>0$ such that $\left(a_{k j}(x)\right) \geq \mu I$ for a.e. $x \in \Omega$, respectively for a.e. $x \in \operatorname{supp} \chi \cup \operatorname{supp} \tilde{\chi}$. In the first case $\Omega$ is a bounded Lipschitz domain of $\mathbb{R}^{d}$ and in the second case $\chi, \widetilde{\chi} \in C_{\mathrm{b}}^{\infty}\left(\mathbb{R}^{d}\right)$. We denote by $S_{t}:=e^{-t A}$ the holomorphic semigroup generated by $-A$ on $L^{2}\left(\mathbb{R}^{d}\right)$. We recall the following result from [12]:

Theorem 3.3. There are $C, c>0$ such that for all $t>0$ the operator $M_{\tilde{\chi}} S_{t} M_{\chi}$ (respectively $P_{\Omega} S_{t} P_{\Omega}$ ) is given by a kernel $p_{t}$ which satisfies

$$
\left|p_{t}(x, y)\right| \leq C t^{-d / 2} e^{-c|x-y|^{2} / t}(1+t)^{d / 2} \quad \text { for all } t>0 \text { and } x, y \in \mathbb{R}^{d} .
$$

The theorem is stated in [12] with $\chi=\tilde{\chi}$, but the arguments work with different $\chi$ and $\tilde{\chi}$. It is also proved there that

$$
\left\|M_{\chi} S_{t}\right\|_{2 \rightarrow \infty} \leq C t^{-d / 4}(1+t)^{d / 4} \quad \text { resp. }\left\|P_{\Omega} S_{t}\right\|_{2 \rightarrow \infty} \leq C t^{-d / 4}(1+t)^{d / 4}
$$

If $z=t+i s \in \mathbb{C}$ with $t=\operatorname{Re} z>0$, then

$$
\begin{aligned}
\left\|M_{\chi} S_{z} M_{\chi}\right\|_{1 \rightarrow \infty} & =\left\|M_{\chi} S_{t / 2} S_{i s} S_{t / 2} M_{\chi}\right\|_{1 \rightarrow \infty} \leq C t^{-d / 4}(1+t)^{d / 4}\left\|S_{i s} S_{t / 2} M_{\chi}\right\|_{1 \rightarrow 2} \\
& \leq C t^{-d / 4}(1+t)^{d / 4}\left\|S_{t / 2} M_{\chi}\right\|_{2 \rightarrow \infty} \leq C t^{-d / 2}(1+t)^{d / 2} .
\end{aligned}
$$


Similarly,

$$
\left\|P_{\Omega} S_{z} P_{\Omega}\right\|_{1 \rightarrow \infty} \leq C(\operatorname{Re} z)^{-d / 2}(1+\operatorname{Re} z)^{d / 2}
$$

for all $z \in \mathbb{C}$ with $\operatorname{Re} z>0$. Using the Gaussian bounds of Theorem 3.3 for real $t$ together with the uniform bounds (3.4) for complex $z$ it follows as in Theorem 3.4.8 in [7] or Theorem 7.2 in [18] that for all $\varepsilon>0$ the kernel $p_{z}^{(0)}$ of $M_{\chi} S_{z} M_{\chi}$, respectively $P_{\Omega} S_{z} P_{\Omega}$, satisfies the bound

$$
\left|p_{z}^{(0)}(x, y) e^{-\varepsilon z}\right| \leq C_{\varepsilon}(\operatorname{Re} z)^{-d / 2} \exp \left\{-c \frac{|x-y|^{2}}{|z|} \cos (\arg z)\right\}
$$

for all $x, y \in \mathbb{R}^{d}$ and $z \in \mathbb{C}$ with $\operatorname{Re} z>0$.

Let $H=A+I$ and define $p_{z}(x, y)=p_{z}^{(0)}(x, y) e^{-z}$. Then $p_{z}$ is the kernel of $M_{\chi} e^{-z H} M_{\chi}$. We shall formulate the results below for $M_{\chi} F(H) M_{\chi}$ only, but all statements are also valid for $P_{\Omega} F(H) P_{\Omega}$. In the following lemmas, we shall always assume that $\left(a_{k j}(x)\right) \geq \mu I$ for almost every $x \in \operatorname{supp} \chi$. Since associated kernels with several operators are involved in the sequel we shall denote by $K_{T}$ the kernel associated to a given operator $T$, whenever it exists.

Lemma 3.4. For all $s>0$ and $\varepsilon>0$ there exists $a C>0$ such that

$$
\int_{\mathbb{R}^{d}}\left|K_{M_{\chi} F(H) M_{\chi}}(x, y)\right|^{2}(1+\sqrt{r}|x-y|)^{s} d x \leq C r^{d / 2}\left\|\delta_{r} F\right\|_{C^{s / 2+\varepsilon}}^{2}
$$

for all $r>0, y \in \mathbb{R}^{d}$ and $F \in C^{s / 2+\varepsilon}$ supported in $[0, r]$. Here $\left(\delta_{r} F\right)(\lambda):=F(r \lambda)$.

Proof. The arguments are very similar to those of Lemma 4.3 in [10]. Fix $r>0$ and assume first that $F$ is supported in $[0,1]$. Set $g(\lambda):=F(\lambda) e^{\lambda}$ and $H_{r}:=\frac{1}{r} H$. By (3.5), the kernel $p_{z / r}$ of $M_{\chi} e^{-z H_{r}} M_{\chi}$ satisfies

$$
\left|p_{z / r}(x, y)\right| \leq C r^{d / 2}(\operatorname{Re} z)^{-d / 2} \exp \left\{-c r \frac{|x-y|^{2}}{|z|} \cos (\arg z)\right\}
$$

for all $x, y \in \mathbb{R}^{d}$ and $z \in \mathbb{C}$ with $\operatorname{Re} z>0$, with constants $C, c$ independent of $r$. We write

$$
g(\lambda)=\int_{\mathbb{R}} \hat{g}(\xi) e^{i \lambda \xi} d \xi,
$$

where $\hat{g}$ is the Fourier transform of $g$. Then

$$
F\left(H_{r}\right)=\int_{\mathbb{R}} \hat{g}(\xi) e^{-(1-i \xi) H_{r}} d \xi
$$

from which one obtains

$$
K_{M_{\chi} F\left(H_{r}\right) M_{\chi}}(x, y)=\int_{\mathbb{R}} \hat{g}(\xi) p_{(1-i \xi) / r}(x, y) d \xi .
$$


Let $y \in \mathbb{R}^{d}$. Using the estimate (3.6) with $z=1-i \xi$ gives

$$
\begin{aligned}
\int_{\mathbb{R}^{d}}\left|p_{(1-i \xi) / r}(x, y)\right|^{2} & (1+\sqrt{r}|x-y|)^{s} d x \\
& \leq C r^{d} \int \exp \left\{-2 c r \frac{|x-y|^{2}}{1+\xi^{2}}\right\}(1+\sqrt{r}|x-y|)^{s} d x \\
& \leq C r^{d}\left(1+\xi^{2}\right)^{s / 2} \int \exp \left\{-c r \frac{|x-y|^{2}}{1+\xi^{2}}\right\} d x \\
& \leq C r^{d}\left(1+\xi^{2}\right)^{s / 2}\left(\frac{1+\xi^{2}}{r}\right)^{d / 2}=C r^{d / 2}\left(1+\xi^{2}\right)^{(d+s) / 2} .
\end{aligned}
$$

It follows from (3.7), the continuous version of the Minkowski inequality, and the previous estimate that

$$
\begin{aligned}
\left(\int_{\mathbb{R}^{d}} \mid K_{M_{\chi} F\left(H_{r}\right) M_{\chi}}\right. & \left.\left.(x, y)\right|^{2}(1+\sqrt{r}|x-y|)^{s} d x\right)^{1 / 2} \\
& \leq \int_{\mathbb{R}}|\hat{g}(\xi)|\left(\int_{\mathbb{R}^{d}}\left|p_{(1-i \xi) / r}(x, y)\right|^{2}(1+\sqrt{r}|x-y|)^{s} d x\right)^{1 / 2} d \xi \\
& \leq C r^{d / 4} \int_{\mathbb{R}}|\hat{g}(\xi)|\left(1+\xi^{2}\right)^{(d+s) / 4} d \xi \\
& \leq C r^{d / 4}\|g\|_{W^{(d+s+2) / 2,2}} \leq C r^{d / 4}\|F\|_{W^{s / 2+\alpha, 2}} .
\end{aligned}
$$

Here $\alpha=(d+2) / 2$ and the constants are independent of $r$ and $y$. On the other hand $M_{\chi} F\left(H_{r}\right) M_{\chi}=M_{\chi} g\left(H_{r}\right) e^{-H_{r}} M_{\chi}$. It follows from (3.3) that

$$
\left\|e^{-H_{r}} M_{\chi}\right\|_{1 \rightarrow 2} \leq C r^{d / 4}\left(1+\frac{1}{r}\right)^{d / 4} e^{-1 / r} \leq C r^{d / 4}
$$

for all $r>0$. Moreover, $\left\|M_{\chi} g\left(H_{r}\right)\right\|_{2 \rightarrow 2} \leq e\|\chi\|_{\infty}\|F\|_{\infty}$. Therefore

$$
\int_{\mathbb{R}^{d}}\left|K_{M_{\chi} F\left(H_{r}\right) M_{\chi}}(x, y)\right|^{2} d x \leq\left\|M_{\chi} F\left(H_{r}\right) M_{\chi}\right\|_{1 \rightarrow 2}^{2} \leq C r^{d / 2}\|F\|_{\infty}^{2} .
$$

This is valid for all $F$ with support in $[0,1]$ and for all $s>0$. The estimates $(3.8)$ and (3.9) together with an interpolation argument (see [17], p. 151, and [10], p. 455) give then that for all $s>0$ there exists a $C>0$ such that

$$
\int_{\mathbb{R}^{d}}\left|K_{M_{\chi} F\left(H_{r}\right) M_{\chi}}(x, y)\right|^{2}(1+\sqrt{r}|x-y|)^{s} d x \leq C r^{d / 2}\|F\|_{C^{s / 2+\varepsilon}}^{2} .
$$

Finally, if $F$ has support in $[0, r]$ we use the last estimate with $\delta_{r} F$ and obtain the lemma.

Lemma 3.5. The operators $A_{t}:=e^{-t^{2} H} M_{\chi}$ satisfy (2.4).

Proof. Let $\psi \in W^{1, \infty}\left(\mathbb{R}^{d}, \mathbb{R}\right)$ be such that $|\nabla \psi| \leq 1$. For all $\rho \in \mathbb{R}$ define $U_{\rho}=$ $M_{e^{\rho \psi}}$ and set $S_{t}^{\rho}:=U_{\rho} e^{-t H} U_{-\rho}$. It follows from [12] Proposition 3.6 by duality and a limit $n \rightarrow \infty$ that there exist $C, \omega>0$, independent of $t, \rho$ and $\psi$, such that

$$
\left\|S_{t}^{\rho} M_{\chi}\right\|_{1 \rightarrow 2} \leq C t^{-d / 4} e^{\omega \rho^{2} t} .
$$


Now fix two bounded open nonempty sets $E$ and $F$ of $\mathbb{R}^{d}$ and choose $\psi(x):=$ $d(x, E) \wedge N$, where $N=\sup \{|x-y|: x \in E, y \in F\}+1$. For all $h \in L^{2}(E)$ and $\rho \geq 0$ one has

$$
M_{\chi} e^{-t H} h=M_{\chi} U_{-\rho} S_{t}^{\rho} h
$$

Therefore

$$
\left\|M_{\chi} e^{-t H} h\right\|_{L^{\infty}(F)} \leq e^{-\rho d(E, F)}\left\|M_{\chi} S_{t}^{\rho} h\right\|_{\infty} \leq C t^{-d / 4} e^{-\rho d(E, F)} e^{\omega \rho^{2} t}\|h\|_{2} .
$$

Choosing $\rho=\frac{d(E, F)}{2 \omega t}$ yields the Davies-Gaffney type estimate

$$
\left\|P_{F}\left(M_{\chi} e^{-t H}\right) P_{E}\right\|_{2 \rightarrow \infty} \leq C t^{-d / 4} e^{-\frac{d(E, F)^{2}}{4 \omega t}} .
$$

In particular,

$$
\left\|P_{C_{j}(x, t)} e^{-t^{2} H} M_{\chi} P_{B(x, t)}\right\|_{1 \rightarrow 2} \leq C t^{-d / 2} e^{-c 4^{j}}
$$

for all $x, y \in \mathbb{R}^{d}$ and $j \in \mathbb{N}$. This shows the lemma.

Proof of Theorems 3.1 and 3.2. As mentioned above, the proofs of both theorems are almost the same. We consider $M_{\chi} F(H) M_{\chi}$ only. The proof is based on Theorem 2.1 and the previous lemmas. It is in the same spirit as in the elliptic case where a Gaussian bound holds (cf. [10], [18]). Let $\varphi \in C_{c}^{\infty}(0, \infty)$ be such that $\operatorname{supp} \varphi \subset[1 / 4,1]$ and

$$
\sum_{n=-\infty}^{\infty} \varphi\left(2^{-n} \lambda\right)=1
$$

for all $\lambda>0$. Then

$$
F(\lambda)=\sum_{n=-\infty}^{\infty} \varphi\left(2^{-n} \lambda\right) F(\lambda)=: \sum_{n=-\infty}^{\infty} F_{n}(\lambda) .
$$

We apply Theorem 2.1 to $M_{\chi} F_{n}(H) M_{\chi}$ for each fixed $n \in \mathbb{Z}$. We choose

$$
S:=M_{\chi} F_{n}(H) \quad \text { and } \quad A_{t}:=e^{-t^{2} H} M_{\chi} .
$$

By Lemma 3.5, the operators $A_{t}$ satisfy (2.4). It remains to prove (2.7). For this we have to estimate for all $y \in \mathbb{R}^{d}$ the integral

$$
I_{n, t}:=\int_{|x-y| \geq t}\left|K_{M_{\chi} G_{n, t}(H) M_{\chi}}(x, y)\right| d x
$$

where

$$
G_{n, t}(\lambda)=F_{n}(\lambda)-F_{n}(\lambda) e^{-t^{2} \lambda}=\varphi\left(2^{-n} \lambda\right) F(\lambda)\left(1-e^{-t^{2} \lambda}\right) .
$$

First, by the Cauchy-Schwarz inequality we have

$$
\begin{aligned}
I_{n, t} \leq & \left(\int_{\mathbb{R}^{d}}\left|K_{M_{\chi} G_{n, t}(H) M_{\chi}}(x, y)\right|^{2}\left(1+2^{n / 2}|x-y|\right)^{2 s} d x\right)^{1 / 2} \\
& \times\left(\int_{|x-y| \geq t}\left(1+2^{n / 2}|x-y|\right)^{-2 s} d x\right)^{1 / 2} .
\end{aligned}
$$


We apply Lemma 3.4 with $r=2^{n}$ and obtain

$$
\int_{\mathbb{R}^{d}}\left|K_{M_{\chi} G_{n, t}(H) M_{\chi}}(x, y)\right|^{2}\left(1+2^{n / 2}|x-y|\right)^{2 s} d x \leq C 2^{n d / 2}\left\|\delta_{2^{n}} G_{n, t}\right\|_{C^{s+\varepsilon}}^{2}
$$

Simple computations show that there exists a $C>0$, independent of $n$ and $t$, such that

$$
\begin{aligned}
\left\|\delta_{2^{n}} G_{n, t}\right\|_{C^{s+\varepsilon}} & =\left\|\varphi(.) F\left(2^{n} \cdot\right)\left(1-e^{-t^{2} 2^{n} \cdot}\right)\right\|_{C^{s+\varepsilon}} \\
& \leq C \sup _{t^{\prime}>0}\left\|\varphi(.) F\left(t^{\prime} .\right)\right\|_{C^{s+\varepsilon}} \min \left(1, t^{2} 2^{n}\right)
\end{aligned}
$$

On the other hand (see [10] or (7.46) in [18]) one estimates

$$
\int_{|x-y| \geq t}\left(1+2^{n / 2}|x-y|\right)^{-2 s} d x \leq C 2^{-n d / 2} \min \left(1,\left(t 2^{n / 2}\right)^{d-2 s}\right) .
$$

Using (3.13), (3.14), (3.15) and (3.16) we obtain

$$
I_{n, t} \leq C \min \left(1, t^{2} 2^{n}\right) \min \left(1,\left(t 2^{n / 2}\right)^{d / 2-s}\right) \sup _{t^{\prime}>0}\left\|\varphi(.) F\left(t^{\prime} .\right)\right\|_{C^{s+\varepsilon}} .
$$

Hence

$$
\sum_{n=-\infty}^{\infty} I_{n, t} \leq C\left(\sum_{n \in \mathbb{Z}, t^{2} 2^{n} \leq 1} t^{2} 2^{n}+\sum_{n \in \mathbb{Z}, t 2^{n / 2}>1}\left(t 2^{n / 2}\right)^{d / 2-s}\right) \sup _{t^{\prime}>0}\left\|\varphi(.) F\left(t^{\prime} .\right)\right\|_{C^{s+\varepsilon}}
$$

and the right hand side is bounded by a constant independent of $t$ since $s>d / 2$. This proves Theorem 3.1 .

As explained in the introduction, the reason why we consider $H=A+I$ instead of $A$ in the previous results comes from the fact the Gaussian upper bound in Theorem 3.3 is valid with the extra factor $(1+t)^{d / 2}$. If one considers the case where $a_{k j}=\delta_{k j}$ on a smooth bounded domain $\Omega$, then $A$ is the Neumann Laplacian on $L^{2}(\Omega)$ and 0 on $L^{2}\left(\mathbb{R}^{d} \backslash \Omega\right)$. It is then easy to see that $L^{2}-L^{\infty}$ estimates (respectively, Gaussian bounds) for $M_{\chi} e^{-t A}$ or $P_{\Omega} e^{-t A}$ (respectively, $M_{\chi} e^{-t A} M_{\chi}$ or $P_{\Omega} e^{-t A} P_{\Omega}$ ) cannot hold without an extra factor $(1+t)^{d / 4}$ (respectively, $\left.(1+t)^{d / 2}\right)$. On the other hand, in the previous theorems we can replace $H=A+I$ by $H=A+\varepsilon I$ for any $\varepsilon>0$.

It may be possible that if $\left(a_{k j}\right) \geq \mu I$ on a connected subset $F$ of $\mathbb{R}^{d}$ which is 'large enough' (in some sense), one can obtain Theorem 3.3 without the extra factor $(1+t)^{d / 2}$ in the Gaussian bound. This remains to be proved. We mention that if such a bound holds, we obtain by the same proof Theorems 3.1 and 3.2 for $F(A)$ rather than $F(H)$.

We emphasize also that we consider here general degenerate operators with nonsmooth coefficients. One may obtain global results for some specific operators which are degenerate at every point and have coefficients that are not continuous at every point. For example, one might take a pure second-order subelliptic operator 
in divergence form with real measurable coefficients on a Lie group with polynomial growth. Then global Gaussian bounds are valid by Théorème 1 of [19], together with a regularization argument (see, for example Section 2.1 in [14]). Therefore a global spectral multiplier result for such operators follows directly from [10]. Note however that the order of smoothness required on the function $F$ is larger than half the Euclidean dimension. On the other hand, the operators that we consider in this paper are allowed to vanish on big sets.

Examples 3.6. We give some examples which are direct applications of the previous theorems.

Imaginary powers. Set $F(\lambda)=\lambda^{i s}$ where $s \in \mathbb{R}$. Then Theorems 3.1 and 3.2, together with the Riesz-Thorin interpolation theorem, imply that for all $\varepsilon>0$ and $p \in(1, \infty)$ there exists a $C>0$ such that

$$
\left\|M_{\chi} H^{i s} M_{\chi}\right\|_{p \rightarrow p} \leq C_{\varepsilon}(1+|s|)^{(d+\varepsilon)|1 / 2-1 / p|}
$$

and

$$
\left\|P_{\Omega} H^{i s} P_{\Omega}\right\|_{\mathcal{L}\left(L^{p}\right)} \leq C_{\varepsilon}(1+|s|)^{(d+\varepsilon)|1 / 2-1 / p|}
$$

for all $s \in \mathbb{R}$.

The Schrödinger group. Set $F(\lambda)=(1+\lambda)^{-\alpha} e^{i t \lambda}$ with $t \in \mathbb{R}$ and $\alpha>d / 2$. The operators $M_{\chi}(I+H)^{-\alpha} e^{i t H} M_{\chi}$ and $P_{\Omega}(I+H)^{-\alpha} e^{i t H} P_{\Omega}$ are bounded on $L^{p}\left(\mathbb{R}^{d}\right)$ for all $p \in(1, \infty)$. Their $L^{p}$-norms are estimated by $C(1+|t|)^{\alpha}$. By interpolation, we obtain boundedness on $L^{p}$ for all $\alpha>d|1 / 2-1 / p|$ and $t \in \mathbb{R}$.

Remark. Using the same proof as in [4], these results can be obtained directly from the Gaussian upper bound of Theorem 3.3 without appealing to Theorems 3.1 and 3.2 .

Wave operators. Set $F(\lambda)=(1+\lambda)^{-\alpha / 2} e^{i t \sqrt{\lambda}}$ with $t \in \mathbb{R}$ and $\alpha>d / 2$. The operators $M_{\chi}(I+H)^{-\alpha} e^{i t \sqrt{H}} M_{\chi}$ and $P_{\Omega}(I+H)^{-\alpha} e^{i t \sqrt{H}} P_{\Omega}$ are bounded on $L^{p}\left(\mathbb{R}^{d}\right)$ for all $p \in(1, \infty)$.

\section{Riesz transforms}

The aim in this section is to prove boundedness on $L^{p}\left(\mathbb{R}^{d}\right)$ of a type of Riesz transform operator $M_{\chi} \nabla(I+A)^{-1 / 2} M_{\chi}$. We keep the same notation as in the previous section. The main result of this section is the next theorem.

Theorem 4.1. Let $\chi \in C_{\mathrm{b}}^{\infty}\left(\mathbb{R}^{d}\right), \mu>0$ and suppose that $\left(a_{k j}(x)\right) \geq \mu I$ for almost every $x \in \operatorname{supp} \chi$. Set $H=A+I$. Then for every $k \in\{1, \ldots, d\}$, the operator $M_{\chi} \partial_{k} H^{-1 / 2} M_{\chi}$ is of weak type $(1,1)$ and is bounded on $L^{p}\left(\mathbb{R}^{d}\right)$ for all $p \in(1,2]$.

Here $\partial_{k}$ denotes the distributional derivative. The proof is based on Theorem 2.1 and uses some ideas from [6], [8], and Chapter 7 in [18] in the uniformly elliptic case. We start with the following lemma. Let $\mathfrak{a}$ be the closure of the regular part of the form $\mathfrak{a}_{0}$ defined in (1.5). 
Lemma 4.2. Let $\chi \in C_{\mathrm{b}}^{\infty}\left(\mathbb{R}^{d}\right), \mu>0$, and suppose that $\left(a_{k j}(x)\right) \geq \mu I$ for almost every $x \in \operatorname{supp} \chi$. Then $\chi u \in W^{1,2}\left(\mathbb{R}^{d}\right)$ and

$$
\left\|\chi \partial_{k} u\right\|_{2}^{2} \leq \frac{\|\chi\|_{\infty}^{2}}{\mu}\left\|H^{1 / 2} u\right\|_{2}
$$

for all $u \in D(\mathfrak{a})=D\left(H^{1 / 2}\right)$ and $k \in\{1, \ldots, d\}$.

Proof. Let $u \in D(\mathfrak{a})$. Then there exists a sequence $\left(u_{n}\right)_{n \in \mathbb{N}}$ in $D\left(\mathfrak{a}_{0}\right)=C_{c}^{\infty}\left(\mathbb{R}^{d}\right)$ such that $\lim u_{n}=u$ in $L^{2}\left(\mathbb{R}^{d}\right)$ and $\mathfrak{a}(u)=\lim \mathfrak{a}_{0}\left(u_{n}\right)$. By the ellipticity assumption on the support of $\chi$ one deduces

$$
\mu \int_{\mathbb{R}^{d}} \chi^{2}\left|\nabla u_{n}\right|^{2} \leq \sum_{k, j=1}^{d} \int_{\mathbb{R}^{d}} a_{k j}\left(\partial_{k} u_{n}\right)\left(\partial_{j} u_{n}\right) \chi^{2} \leq\|\chi\|_{\infty}^{2} \mathfrak{a}_{0}\left(u_{n}\right)
$$

for all $n \in \mathbb{N}$. Therefore $\left(\chi u_{n}\right)_{n \in \mathbb{N}}$ is bounded in $W^{1,2}\left(\mathbb{R}^{d}\right)$. Hence it has a weakly convergent subsequence in $W^{1,2}\left(\mathbb{R}^{d}\right)$. Since $\lim \chi u_{n}=\chi u$ in $L^{2}\left(\mathbb{R}^{d}\right)$ it follows that $\chi u \in W^{1,2}\left(\mathbb{R}^{d}\right)$. Then taking the limit $n \rightarrow \infty$ in (4.1) one estimates

$$
\mu \int_{\mathbb{R}^{d}} \chi^{2}\left|\partial_{k} u\right|^{2} \leq\|\chi\|_{\infty}^{2} \mathfrak{a}(u) \leq\|\chi\|_{\infty}^{2}\left\|H^{1 / 2} u\right\|_{2}^{2}
$$

for all $k \in\{1, \ldots, d\}$.

Lemma 4.3. Let $\chi, \tilde{\chi} \in C_{\mathrm{b}}^{\infty}\left(\mathbb{R}^{d}\right), \mu>0$, and assume that $\left(a_{k j}(x)\right) \geq \mu I$ for almost every $x \in \operatorname{supp} \chi \cup \operatorname{supp} \tilde{\chi}$. Then for all $\beta>0$ small enough we have

$$
\int_{\mathbb{R}^{d}}\left|\left(M_{\widetilde{\chi}} e^{-s H} M_{\chi} u\right)(y)\right|^{2} e^{\beta|x-y|^{2} / s} d y \leq C s^{-d / 2} e^{2 \beta t^{2} / s} e^{-s}\|u\|_{1}^{2}
$$

for all $t>0, s>0, x \in \mathbb{R}^{d}$ and $u \in L^{2}\left(\mathbb{R}^{d}\right)$ with $\operatorname{supp} u \subset B(x, t)$.

Proof. By Theorem 3.3 one estimates

$$
\begin{aligned}
\mid\left(M_{\tilde{\chi}} e^{-s H}\right. & \left.M_{\chi} u\right)\left.(y)\right|^{2} e^{\beta|x-y|^{2} / s}=\left|\int_{B(x, t)} e^{-s} p_{s}(y, z) u(z) d z\right|^{2} e^{\beta|x-y|^{2} / s} \\
& \leq C e^{-s}\left(\int_{B(x, t)} s^{-d / 2} e^{-c|y-z|^{2} / s} e^{\beta|x-y|^{2} /(2 s)}|u(z)| d z\right)^{2} \\
& \leq C e^{-s}\left(\int_{\mathbb{R}^{d}} s^{-d / 2} e^{-(c-\beta)|y-z|^{2} / s}|u(z)| d z\right)^{2} e^{2 \beta t^{2} / s} \\
& \leq C e^{-s} s^{-d / 2}\|u\|_{1} \int_{\mathbb{R}^{d}} s^{-d / 2} e^{-(c-\beta)|y-z|^{2} / s}|u(z)| d z e^{2 \beta t^{2} / s} .
\end{aligned}
$$

Taking $\beta<c / 2$ and integrating over $y$ yields the lemma.

Since $e^{-s H} L^{2}\left(\mathbb{R}^{d}\right) \subset D(\mathfrak{a})$ for all $s>0$ we obtain from Lemma 4.2 the inclusion $M_{\chi} \nabla e^{-s H} M_{\chi}\left(L^{2}\left(\mathbb{R}^{d}\right)\right) \subset W^{1,2}\left(\mathbb{R}^{d}\right)$ for all $s>0$. The following weighted $L^{2}$-estimate is in the same spirit as weighted gradient estimates for heat kernels (see [6], [15] and Theorem 6.19 in [18]). 
Lemma 4.4. For all $\beta>0$ small enough we have

$$
\int_{\mathbb{R}^{d}}\left|\left(M_{\chi} \nabla e^{-s H} M_{\chi} u\right)(y)\right|^{2} e^{\beta|x-y|^{2} / s} d y \leq C s^{-d / 2-1} e^{6 \beta t^{2} / s}\|u\|_{1}^{2}
$$

for all $t>0, s>0, x \in \mathbb{R}^{d}$ and $u \in L^{2}\left(\mathbb{R}^{d}\right)$ with $\operatorname{supp} u \subset B(x, t)$.

Proof. In order to avoid problems related to the domain of forms, we shall proceed by approximation. First, we prove the lemma for uniformly elliptic coefficients with constants $\beta$ and $C$ depending only on $\mu>0$ such that $\left(a_{k j}(x)\right) \geq \mu I$ a.e. $x \in \operatorname{supp} \chi$.

Assume that there exists a $\mu_{0}>0$ such that $\left(a_{k j}(x)\right) \geq \mu_{0} I$ for a.e. $x \in \mathbb{R}^{d}$. In this case the form $\mathfrak{a}$ has domain $W^{1,2}\left(\mathbb{R}^{d}\right)$. We use ideas similar to those in the proof of Theorem 6.19 in [18], but we want to prove that the constants in the estimates are independent of $\mu_{0}$. Let $\psi \in C_{c}^{\infty}\left(\mathbb{R}^{d}\right)$ be such that $\psi(x)=1$ for all $x \in B(0,1)$ and $0 \leq \psi \leq \mathbb{1}$. For all $n \in \mathbb{N}$, define $\psi_{n} \in C_{c}^{\infty}\left(\mathbb{R}^{d}\right)$ by $\psi_{n}(x)=\psi\left(n^{-1} x\right)$. Set

$$
I_{n}:=\int_{\mathbb{R}^{d}}\left|\chi(y)\left(\nabla e^{-s H} M_{\chi} u\right)(y)\right|^{2} e^{\beta|x-y|^{2} / s} \psi_{n}(y) d y
$$

and define $f:=e^{-s H} M_{\chi} u$. Then,

$$
\begin{aligned}
I_{n} \leq & \frac{1}{\mu} \sum_{k, j} \int_{\mathbb{R}^{d}} a_{k j}(y)\left(\partial_{k} f\right)(y)\left(\partial_{j} f\right)(y) e^{\beta|x-y|^{2} / s} \chi(y)^{2} \psi_{n}(y) d y \\
= & \frac{1}{\mu} \sum_{k, j} \int_{\mathbb{R}^{d}} a_{k j}\left(\partial_{k} f\right) \partial_{j}\left(f e^{\beta|x-\cdot|^{2} / s} \chi^{2} \psi_{n}\right) \\
& +\frac{1}{\mu} \sum_{k, j} \int_{\mathbb{R}^{d}} a_{k j}(y)\left(\partial_{k} f\right)(y) f(y) \frac{2 \beta\left(x_{j}-y_{j}\right)}{s} e^{\beta|x-y|^{2} / s} \chi(y)^{2} \psi_{n}(y) d y \\
& -\frac{2}{\mu} \sum_{k, j} \int_{\mathbb{R}^{d}} a_{k j}(y)\left(\partial_{k} f\right)(y) f(y) e^{\beta|x-y|^{2} / s}\left(\partial_{j} \chi\right)(y) \chi(y) \psi_{n}(y) d y \\
& -\frac{1}{n \mu} \sum_{k, j} \int_{\mathbb{R}^{d}} a_{k j}(y)\left(\partial_{k} f\right)(y) f(y) e^{\beta|x-y|^{2} / s} \chi(y)^{2}\left(\partial_{j} \psi\right)\left(\frac{1}{n} y\right) d y \\
= & : J_{1, n}+J_{2, n}+J_{3, n}+J_{4, n} .
\end{aligned}
$$

Since $y \mapsto f(y) e^{\beta \frac{|x-y|^{2}}{s}} \chi(y)^{2} \psi_{n}(y)$ is an element of $W^{1,2}\left(\mathbb{R}^{d}\right)$ we have

$$
\begin{aligned}
J_{1, n} & =\frac{1}{\mu} \mathfrak{a}\left(f, f e^{\beta|x-\cdot|^{2} / s} \chi^{2} \psi_{n}\right)=\frac{1}{\mu} \int_{\mathbb{R}^{d}}\left(A e^{-s H} M_{\chi} u\right)\left(e^{-s H} M_{\chi} u\right) e^{\beta|x-\cdot|^{2} / s} \chi^{2} \psi_{n} \\
& \leq \frac{\|\chi\|_{\infty}}{\mu}\left\|H e^{-s H} M_{\chi} u\right\|_{2}\left\|e^{\beta|x-\cdot|^{2} / s} M_{\chi} e^{-s H} M_{\chi} u\right\|_{2} .
\end{aligned}
$$

The standard estimate $\left\|H e^{-s H}\right\|_{2 \rightarrow 2} \leq s^{-1}$ and Lemma 4.3 give

$$
J_{1, n} \leq C s^{-d / 2-1} e^{2 \beta t^{2} / s}\|u\|_{1}^{2}
$$


if $\beta$ is small enough. Using the obvious inequality $\frac{\left|x_{j}-y_{j}\right|}{s} \leq \frac{1}{\sqrt{\varepsilon s}} e^{\varepsilon \frac{|x-y|^{2}}{s}}$ we have

$$
\begin{aligned}
\left|J_{2, n}\right| & \leq \frac{C}{\sqrt{s}} \sum_{k} \int_{\mathbb{R}^{d}}\left|\partial_{k} e^{-s H} M_{\chi} u\right| \chi^{2}\left|e^{-s H} M_{\chi} u\right| e^{2 \beta|x-\cdot|^{2} / s} \\
& \leq \frac{C}{\sqrt{s}} \sqrt{I_{n}}\left(\int_{\mathbb{R}^{d}}\left|\left(M_{\chi} e^{-s H} M_{\chi} u\right)(y)\right|^{2} e^{3 \beta|x-y|^{2} / s} d y\right)^{1 / 2} .
\end{aligned}
$$

Therefore Lemma 4.3 implies

$$
\left|J_{2, n}\right| \leq C \sqrt{I_{n}} s^{-d / 4-1 / 2} e^{-s / 2} e^{3 \beta t^{2} / s}\|u\|_{1} .
$$

We estimate the third term in a similar way.

$$
\begin{aligned}
\left|J_{3, n}\right| & \leq C \sum_{k} \int_{\mathbb{R}^{d}}\left|\partial_{k} e^{-s H} M_{\chi} u\right|\left|\chi \partial_{j} \chi\right|\left|e^{-s H} M_{\chi} u\right| e^{\beta|x-\cdot|^{2} / s} \psi_{n} \\
& \leq C \sqrt{I_{n}}\left(\int_{\mathbb{R}^{d}}\left|M_{\partial_{j} \chi} e^{-s H} M_{\chi} u\right|^{2} e^{\beta|x-\cdot|^{2} / s}\right)^{1 / 2} \\
& \leq C \sqrt{I_{n}} s^{-d / 4-1 / 2} e^{-s / 3} e^{\beta t^{2} / s}\|u\|_{1} .
\end{aligned}
$$

Finally,

$$
\begin{aligned}
\left|J_{4, n}\right| \leq & \frac{C}{n} \sum_{k, j} \int_{\mathbb{R}^{d}}\left|\left(\chi \partial_{k} e^{-s H} M_{\chi} u\right)(y)\right|\left|\left(M_{\chi} e^{-s H} M_{\chi} u\right)(y)\right| e^{\beta|x-y|^{2} / s} \\
& \times\left|\left(\partial_{j} \psi\right)\left(\frac{1}{n} y\right)\right| d y \\
\leq & \frac{C}{n}\left\|M_{\chi} \nabla e^{-s H} M_{\chi} u\right\|_{2}\left(\int_{\mathbb{R}^{d}}\left|\left(M_{\chi} e^{-s H} M_{\chi} u\right)(y)\right|^{2} e^{2 \beta|x-y|^{2} / s} d y\right)^{1 / 2} \\
\leq & \frac{C}{n}\left\|M_{\chi} \nabla e^{-s H} M_{\chi} u\right\|_{2} s^{-d / 4} e^{-s / 2} e^{2 \beta t^{2} / s}\|u\|_{1} .
\end{aligned}
$$

Therefore, we obtain from $(4.2),(4.3),(4.4)$ and (4.5) that

$$
I_{n} \leq C s^{-d / 2-1} e^{6 \beta t^{2} / s}\|u\|_{1}^{2}+\frac{C}{n}\left\|M_{\chi} \nabla e^{-s H} M_{\chi} u\right\|_{2} s^{-d / 4} e^{2 \beta t^{2} / s}\|u\|_{1} .
$$

Letting $n \rightarrow \infty$ and then using Fatou's lemma yields

$$
\int_{\mathbb{R}^{d}}\left|\left(M_{\chi} \nabla e^{-s H} M_{\chi} u\right)(y)\right|^{2} e^{\beta|x-y|^{2} / s} d y \leq C s^{-d / 2-1} e^{6 \beta t^{2} / s}\|u\|_{1}^{2} .
$$

The constants $C$ and $\beta$ are independent of $\mu_{0}$.

Now we prove the lemma for degenerate operators. For all $n \in \mathbb{N}$ set $a_{k j}^{(n)}=$ $a_{k j}+\delta_{k j} / n$. Then $\left(a_{k j}^{(n)}(x)\right) \geq \frac{1}{n} I$ for a.e. $x \in \mathbb{R}^{d}$ and $\left(a_{k j}^{(n)}(x)\right) \geq \mu I$ for a.e. $x \in \operatorname{supp} \chi$. Moreover, $\left\|a_{k j}^{(n)}\right\|_{\infty} \leq 1+\left\|a_{k j}\right\|_{\infty}$. We denote by $A_{n}$ the elliptic operator with the coefficients $a_{k j}^{(n)}$ and let $H_{n}=I+A_{n}$. We apply (4.6) to $H_{n}$ and obtain

$$
\int_{\mathbb{R}^{d}}\left|\left(M_{\chi} \nabla e^{-s H_{n}} M_{\chi} u\right)(y)\right|^{2} e^{\beta|x-y|^{2} / s} d y \leq C s^{-d / 2-1} e^{6 \beta t^{2} / s}\|u\|_{1}^{2}
$$


for some constants $C$ and $\beta>0$ which are independent of $n$. Let $k \in\{1, \ldots, d\}$. Then

$$
\left|\left(e^{-s H_{n}} M_{\chi} u, \partial_{k} M_{\chi}\left(\varphi e^{\beta \frac{|x-.|^{2}}{2 s}}\right)\right)\right| \leq C s^{-d / 4-1 / 2} e^{3 \beta t^{2} / s}\|u\|_{1}\|\varphi\|_{2}
$$

for all $\varphi \in C_{c}^{\infty}\left(\mathbb{R}^{d}\right)$. On the other hand, $e^{-t H_{n}}$ converges strongly in $L^{2}\left(\mathbb{R}^{d}\right)$ to $e^{-t H}$ (see Corollary 3.9 of [1]). It follows then that

$$
\left|\left(e^{-s H} M_{\chi} u, \partial_{k} M_{\chi}\left(\varphi e^{\beta \frac{\left\lfloor x-\left.\right|^{2}\right.}{2 s}}\right)\right)\right| \leq C s^{-d / 4-1 / 2} e^{3 \beta t^{2} / s}\|u\|_{1}\|\varphi\|_{2} .
$$

Since this is true for all $\varphi \in C_{c}^{\infty}\left(\mathbb{R}^{d}\right)$ we have by density

$$
\left\|\left(M_{\chi} \partial_{k} e^{-s H} M_{\chi} u\right) \cdot e^{\beta \frac{|x-.|^{2}}{2 s}}\right\|_{2} \leq C s^{-d / 4-1 / 2} e^{3 \beta t^{2} / s}\|u\|_{1} .
$$

This proves the lemma.

Proof of Theorem 4.1. It follows from Lemma 4.2 that the truncated Riesz transform $M_{\chi} \partial_{k} A^{-1 / 2}$ is bounded on $L^{2}\left(\mathbb{R}^{d}\right)$.

In order to prove a weak type estimate for $T=M_{\chi} \partial_{k} H^{-1 / 2} M_{\chi}$ we apply Theorem 2.1 with $S=M_{\chi} \partial_{k} H^{-1 / 2}$ and $A_{t}=e^{-t^{2} H} M_{\chi}$. These operators are bounded on $L^{2}\left(\mathbb{R}^{d}\right)$ and by Lemma 3.5 the operators $A_{t}$ satisfy assumption (2.4). It remains then to check (2.5). By the formula

$$
H^{-1 / 2}=\frac{1}{2 \sqrt{\pi}} \int_{0}^{\infty} e^{-s H} \frac{d s}{\sqrt{s}}
$$

we have

$$
H^{-1 / 2} e^{-t^{2} H}=\frac{1}{2 \sqrt{\pi}} \int_{0}^{\infty} e^{-\left(s+t^{2}\right) H} \frac{d s}{\sqrt{s}}=\frac{1}{2 \sqrt{\pi}} \int_{0}^{\infty} e^{-s H} \mathbb{1}_{\left\{s>t^{2}\right\}} \frac{d s}{\sqrt{s-t^{2}}} .
$$

Let $\beta>0$ be as in Lemma 4.4 and let $\delta>0$. Fix $x \in \mathbb{R}^{d}, t>0$ and let $u \in L^{2}\left(\mathbb{R}^{d}\right)$ with supp $u \subset B(x, t)$. Set

$$
\nu(s, t)=\left|\mathbb{1}_{\left\{s>t^{2}\right\}} \frac{1}{\sqrt{s-t^{2}}}-\frac{1}{\sqrt{s}}\right| .
$$

Then

$$
\begin{aligned}
2 \sqrt{\pi} & \int_{\mathbb{R}^{d} \backslash B(x,(1+\delta) t)}\left|\left(\left(T-S A_{t}\right) u\right)(y)\right| d y \\
& \leq \int_{0}^{\infty} \int_{\mathbb{R}^{d} \backslash B(x,(1+\delta) t)}\left|\left(M_{\chi} \partial_{k} e^{-s H} M_{\chi} u\right)(y)\right| d y \nu(s, t) d s \\
& \leq \int_{0}^{\infty} \nu(s, t)\left(\int_{\mathbb{R}^{d}}\left|\left(M_{\chi} \partial_{k} e^{-s H} M_{\chi} u\right)(y)\right|^{2} e^{\beta|x-y|^{2} / s} d y\right)^{1 / 2} \\
& \times\left(\int_{\mathbb{R}^{d} \backslash B(x,(1+\delta) t)} e^{-\beta|x-y|^{2} / s} d y\right)^{1 / 2} d s \\
& \leq C \int_{0}^{\infty} \nu(s, t) s^{-d / 4-1 / 2} e^{3 \beta t^{2} / s}\|u\|_{1}\left(\int_{\mathbb{R}^{d} \backslash B(x,(1+\delta) t)} e^{-\beta|x-y|^{2} / s} d y\right)^{1 / 2} d s .
\end{aligned}
$$


Note that we have used Lemma 4.4 in the last inequality. Now

$$
\begin{aligned}
\int_{\mathbb{R}^{d} \backslash B(x,(1+\delta) t)} e^{-\beta|x-y|^{2} / s} d y & \leq e^{-\beta(1+\delta)^{2} t^{2} /(2 s)} \int_{\mathbb{R}^{d}} e^{-\beta|x-y|^{2} /(2 s)} d y \\
& \leq C s^{d / 2} e^{-\beta(1+\delta)^{2} t^{2} /(2 s)} .
\end{aligned}
$$

Choosing $\delta \geq 4$ we obtain a positive constant $\gamma$ such that

$$
\int_{\mathbb{R}^{d} \backslash B(x,(1+\delta) t)}\left|\left(T-S A_{t}\right) u(y)\right| d y \leq C \int_{0}^{\infty} \nu(s, t) s^{-1 / 2} e^{-\gamma t^{2} / s} d s .
$$

The last integral is bounded by some constant $M$ independent of $t$. This proves the estimate (2.5) and hence $T=M_{\chi} \partial_{k} H^{-1 / 2} M_{\chi}$ is weak type $(1,1)$. By interpolation, it is bounded on $L^{p}\left(\mathbb{R}^{d}\right)$ for all $1<p \leq 2$.

As discussed at the end of the previous section, we note that if one proves a version of Theorem 3.3 without the extra factor $(1+t)^{d / 2}$ if $\left(a_{k j}(x)\right) \geq \mu I$ for a.e. $x$ in a 'big' domain, then Theorem 4.1 holds with $A$ in place of $H$. That is $M_{\chi} \partial_{k} A^{-1 / 2} M_{\chi}$ is weak type $(1,1)$ and bounded on $L^{p}\left(\mathbb{R}^{d}\right)$ for all $1<p \leq 2$. In [13], we prove by a different method that if the coefficients $a_{k j} \in W^{1, \infty}\left(\mathbb{R}^{d}\right)$, then $M_{\chi} \partial_{k}(I+A)^{-1 / 2}$ and $M_{\chi} \partial_{k} \partial_{j}(I+A)^{-1}$ are bounded on $L^{p}\left(\mathbb{R}^{d}\right)$ for all $p \in$ $(1, \infty)$. Moreover, if $a_{k j} \in W^{\nu, \infty}\left(\mathbb{R}^{d}, \mathbb{C}\right)$ then we show that $M_{\chi} \partial_{k}(I+A)^{-1 / 2} M_{\chi}$ is bounded on $L^{p}$ for all $p \in(1, \infty)$.

\section{References}

[1] Arendt, W. and ter Elst, A. F. M.: Sectorial forms and degenerate differential operators. J. Operator Theory 67 (2012), no. 1, 33-72.

[2] Auscher, P.: On necessary and sufficient conditions for $L^{p}$-estimates of Riesz transforms associated to elliptic operators on $\mathbb{R}^{n}$ and related estimates. Mem. Amer. Math. Soc. 186 (2007), no. 871, xviii+75 pp.

[3] Blunck, S. and Kunstmann, P. C.: Calderón-Zygmund theory for non-integral operators and the $H^{\infty}$-functional calculus. Rev. Mat. Iberoamericana 19 (2003), no. 3, 919-942.

[4] Carron, G., Coulhon, T. and Ouhabaz, E. M.: Gaussian estimates and $L_{p}$-boundedness of Riesz means. J. Evol. Equ. 2 (2002), no. 3, 299-317.

[5] Coifman, R. R. And Weiss, G.: Analyse harmonique non-commutative sur certains espaces homogénes. Lecture Notes in Mathematics 242, Springer-Verlag, Berlin-New York, 1971.

[6] Coulhon, T. And Duong, X. T.: Riesz transforms for $1 \leq p \leq 2$. Trans. Amer. Math. Soc. 351 (1999), no. 3, 1151-1169.

[7] Davies, E. B.: Heat kernels and spectral theory. Cambridge Tracts in Mathematics 92, Cambridge University Press, Cambridge, 1989.

[8] Duong, X. T. and McIntosh, A.: The $L^{p}$ boundedness of Riesz transforms associated with divergence form operators. In Joint Australian-Taiwanese workshop on analysis and applications (Brisbane, 1997), 15-25. Proc. Centre Math. Anal. and its Appl. 37, 1999. 
[9] Duong, X. T. And McIntosh, A.: Singular integral operators with non-smooth kernels on irregular domains. Rev. Mat. Iberoamericana 15 (1999), no. 2, 233-265.

[10] Duong, X. T., Ouhabaz, E. M. And Sikora, A.: Plancherel-type estimates and sharp spectral multipliers. J. Funct. Anal. 196 (2002), no. 2, 443-485.

[11] Duong, X. T. And Yan, L.: Duality of Hardy and BMO spaces associated with operators with heat kernel bounds. J. Amer. Math. Soc. 18 (2005), no. 4, 943-973.

[12] ter Elst, A. F. M. And Ouhabaz, E. M.: Partial Gaussian bounds for degenerate differential operators. Potential Anal. 35 (2011), no. 2, 175-199.

[13] ter Elst, A.F. M. And Ouhabaz, E. M.: Partial Gaussian bounds for degenerate differential operators II. To appear in Ann. Sc. Norm. Super. Pisa Cl. Sci.

[14] ter Elst, A.F. M. And Robinson, D. W.: Second-order subelliptic operators on Lie groups. I. Complex uniformly continuous principal coefficients. Acta Appl. Math. 59 (1999), no. 3, 299-331.

[15] Grigor'yan, A.: Upper bounds of derivatives of the heat kernel on an arbitrary complete manifold. J. Funct. Anal. 127 (1995), no. 2, 363-389.

[16] Hofmann, S., Lu, G., Mitrea, D., Mitrea, M. and Yan, L.: Hardy spaces associated to non-negative self-adjoint operators satisfying Davies-Gaffney estimates. Mem. Amer. Math. Soc. 214 (2011), no. 1007, vi+78 pp.

[17] Mauceri, M. and Meda, S.: Vector-valued multipliers on stratified groups. Rev. Mat. Iberoamericana 6 (1990), no. 3-4, 141-154.

[18] Ouhabaz, E. M.: Analysis of heat equations on domains. London Mathematical Society Monographs Series 31, Princeton University Press, Princeton, NJ, 2005.

[19] Saloff-Coste, L. And Stroock, D. W.: Opérateurs uniformément sous-elliptiques sur les groupes de Lie. J. Funct. Anal. 98 (1991), no. 1, 97-121.

[20] Shen, Z.: Bounds of Riesz transforms on $L^{p}$ spaces for second order elliptic operators. Ann. Inst. Fourier (Grenoble) 55 (2005), no. 1, 173-197.

[21] Simon, B.: A canonical decomposition for quadratic forms with applications to monotone convergence theorems. J. Funct. Anal. 28 (1978), no. 3, 377-385.

[22] Stein, E. M.: Harmonic analysis: real-variable methods, orthogonality, and oscillatory integrals. Princeton Mathematical Series 43, Monographs in Harmonic Analysis III, Princeton University Press, Princeton, NJ, 1993.

Received August 11, 2011; revised January 5, 2012.

A. F. M. TER Elst: Department of Mathematics, University of Auckland, Private bag 92019, Auckland, New Zealand.

E-mail: terelst@math.auckland.ac.nz

E. M. Ouhabaz: Univ. Bordeaux, IMB, CNRS UMR 5251, 351, Cours de la Libération, 33405 Talence, France.

E-mail: Elmaati.Ouhabaz@math.u-bordeaux1.fr

Most of this work was carried out whilst the second named author was visiting the Department of Mathematics at the University of Auckland. He wishes to thank both the University of Auckland and the CNRS for financial support. Part of this work is supported by the Marsden Fund Council from Government funding administered by the Royal Society of New Zealand. 